Research Article

\title{
Prediction and Evaluation Model of Physical Training for Volleyball Players' Effect Based on Grey Markov Theory
}

\author{
Chengcheng Guo \\ Department of Public Education, Anhui Finance \& Trade Vocational College, Hefei, Anhui, China \\ Correspondence should be addressed to Chengcheng Guo; gcc@afc.edu.cn
}

Received 9 August 2021; Revised 6 September 2021; Accepted 8 September 2021; Published 15 September 2021

Academic Editor: Mian Ahmad Jan

Copyright (C) 2021 Chengcheng Guo. This is an open access article distributed under the Creative Commons Attribution License, which permits unrestricted use, distribution, and reproduction in any medium, provided the original work is properly cited.

\begin{abstract}
Physical competition is becoming the new focus of volleyball in an increasingly perfect technical and tactical system. Unfortunately, poor physical fitness is a recognized weakness of volleyball players and a critical factor that has restricted the rapid development of volleyball for a long time. This paper proposes a grey Markov model-based approach to improve the evaluation ability of physical training. It aims to construct an empirical analysis model by combining statistical results and analyzing the evaluation parameters for physical training effects. The sports parameter analysis method is adopted to establish an optimal model of these parameters. Finally, a distribution model of moments of inertia combined with fuzzy information fusion's feature extraction method is proposed for the distributed reconstruction of physical training. The optimization of training effects based on parameter optimization and construction of a grey Markov model enhances the physical training of volleyball players.
\end{abstract}

\section{Introduction}

Due to the socialization and commercialization of competitive sports, more and more countries have begun to invest a lot of manpower and material resources to develop their competitive sports, resulting in increasingly fierce competition in the world sports arena. At present, action technology has reached a relatively mature level. In various competitions, the tactical system composed of multiple action techniques has also been flourished. The publication of different teaching materials and monographs, the current timely reports of various media, and the dissemination of information on the Internet and their respective skills and tactics are not a secret. Therefore, physical fitness has become a particularly prominent factor in winning in sports competitions and achieving excellent sports performance. It can be seen from the competitions of various sports that the situation of relying solely on technical and tactical advantages to win the game is no longer comparable to the past [1-4]. Athletes or teams with superior skills and tactics cannot win the final competition, often due to physical fitness, the shortcomings, especially when the level is exceptionally high. Although volleyball belongs to the skill-led competition of the net, it is also facing this situation. With more and more countries paying attention to volleyball, the current volleyball game has broken the original monopoly, showing a more intense situation of solid competition. With the rapid development of the world volleyball technical level and the continuous modification and improvement of competition rules, modern volleyball technology develops towards higher serve points, diversified cushioning, passing speed, spiking power, and blocking cavitation. Especially, in recent years, its development momentum has become even fiercer [5-8]. This development trend of modern volleyball technology has increasingly made physical fitness a critical factor in winning.

For a volleyball team to achieve excellent sports performance, it must achieve a highly coordinated development of physical fitness, intelligence, tactics, technology, and psychology, as shown in Figure 1. Among the five competitive ability elements, physical fitness is the foundation as good physical fitness will provide the premise and possibility for the full use of skills and tactics [9-14]. Physical fitness is closely related to the application effects of skills and tactics. Today, with the increasingly complete technical and tactical system, physical fitness competition is becoming the new 


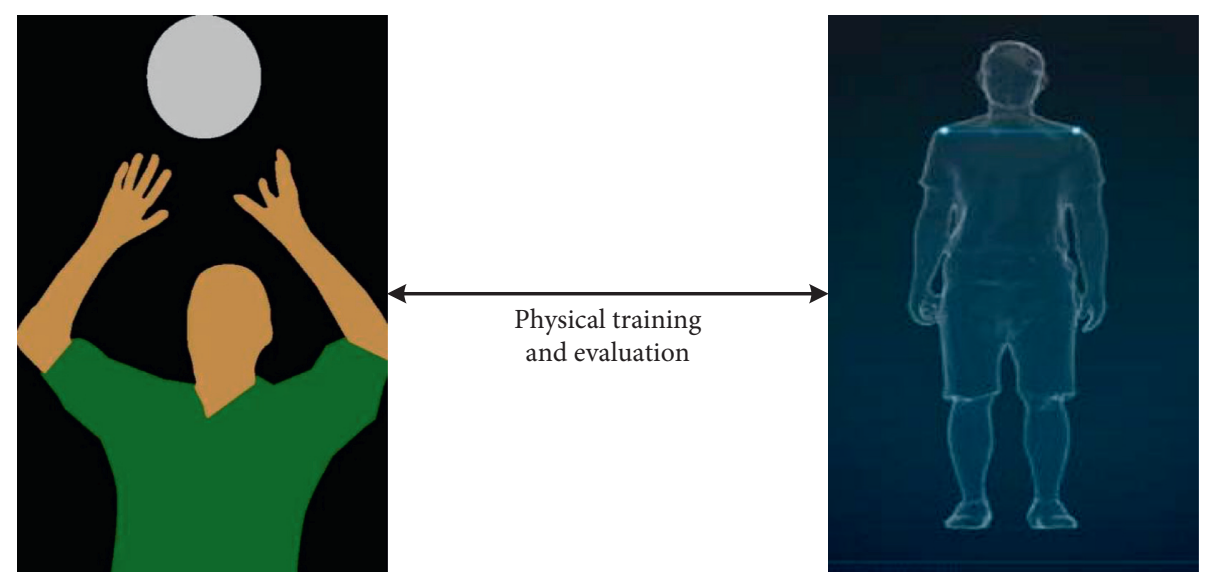

FIgURE 1: Volleyball and physical fitness assessment.

focus of volleyball competitions. Modern volleyball is developing in the direction of fast-paced and intense confrontation, and this fast-paced and strong confrontation requires good physical fitness as a guarantee. Therefore, strengthening the special physical training and improving the athletes' special strength level, jumping ability, and fast movement ability will positively affect the application of skills and tactics in the competition.

With the continuous development of intelligent sports training technology, intelligent digital analysis and quantitative analysis methods are used to evaluate athletes' physical training effects. This approach is combined with parameter optimization and big data analysis techniques to build a big data analysis model for assessing athletes' physical training effects [15-19]. Furthermore, the proposed approach is combined with information fusion to improve the accuracy of evaluation and study various models of athletes' physical training effects, which is of great significance in promoting the optimization of their training [20-25]. We combine the grey theory and Markov theory to formulate a grey Markov physical training effect prediction and evaluation model for volleyball players. This combination can reveal the general trend of physical training of these players. Our model has the ability to reflect the development trend of the data sequence and can also reduce the range of prediction interval via the transformation of state transition probability matrix. Hence, the prediction is more adaptable and the accuracy is much higher.

The rest of this paper is organized as follows. In Section 2 , the related work is presented. In Section 3, the proposed prediction and evaluation model of physical training effect is discussed. In Section 4, the model is tested and discussion of experimental results is provided. Finally, the paper is concluded and future research directions are provided in Section 5.

\section{Related Work}

The evaluation standard is the scale that measures the evaluation objects to meet the requirements of any evaluation index. Therefore, the establishment of scientific and reasonable evaluation standards is of great significance for improving the quality of evaluation. At present, the relevant data related to the volleyball physical fitness evaluation model and standard are summarized as follows. Fu and Cheng [26] put forward the concept of volleyball index, which is used to measure the relationship between the height of volleyball players, the height of the jump, and the height of net in the training competition. Wang [27] conducted an efficient comprehensive multi-index evaluation of the physical fitness of Chinese juvenile volleyball players. Using the percentile system and the average \pm 4 times the standard deviation, they formulated 13 individual scoring standards for physical fitness, and two comprehensive evaluation regression equations are established using the stepwise regression method. Chen [28] established a mathematical model that distinguishes the physical fitness of volleyball players. The 100-meter run that affects the physical fitness is followed by five-level leapfrogging, approach run, 800-meter run, sloping abdomen, and 6-meter run. Yang [29] reprinted the American Men's Volleyball players' Physical Fitness Test Evaluation Form, which included a total of 16 evaluation indicators such as height, single finger height, vertical jump, and block jump, using a 30-point system. Zhang et al. [30] used the progressive scoring method to determine the six physical fitness features of volleyball players, i.e., walk-up touch, 100-meter run, five-level leapfrogging, sloping abdomen, weight-bearing back muscles, and 6-meter movement. It also uses the deviation method to establish the individual and comprehensive physical fitness evaluation standards of athletes (excellent, good, medium, passing, and poor five-level evaluation form). Feng and Mei [31] established the physical fitness evaluation model of different groups of athletes based on special physical fitness of juvenile volleyball players. Yang et al. [32] and Tang et al. [33] established the 100-meter run, 60-meter run, 800-meter run, 1500-meter run, 36-meter movement, the approach height, and the supine abdomen, among others. The physical fitness test evaluation standard conducted by the Chinese Volleyball League also adopts the 100-point system.

In training, coaches and scientific research assistants must pay attention to obtain various feedback information about athletes training, so as to better control the whole 
training process and make training more systematic. Obtaining training information is accomplished through monitoring measures, and then, the acquired information is processed to obtain valuable reference materials to guide future training practices. This processing is essentially the standard procedure for diagnosis. Due to the long-term nature of the physical training process and the complexity of influencing factors, coaches and scientific researchers are required to continuously monitor and evaluate the athlete's training status and make timely adjustments to the training content and methods. With the rapid development of modern science and technology, the methods of training and monitoring have become more advanced and the effects have become more and more obvious. As a result, many countries have established "comprehensive monitoring systems for advanced athletes" for some key projects, so as to achieve effective control on the basis of systematic procedures and have achieved good results. For example, Italy, the Netherlands, Germany, and other European countries use advanced computer technology to quantitatively monitor the physical training process, especially the quantitative evaluation and control of training load, which makes the regulation of the physical training process more scientific. To date, the data on monitoring and diagnostic evaluation of physical training for volleyball has not yet been seen, but there have been beneficial attempts in physical training for other sports in China. Jiang and Chen [34] developed a monitoring and analysis system for an efficient athlete' physical fitness and skills, which has four main functions: data entry and performance conversion, vertical and horizontal analysis, result output, and decision-making plans. In this article, the author puts forward three suggestions for further improvement of the system in the future: increase horizontal comparative analysis, increase intelligent functions, and increase comprehensive evaluation functions.

\section{Prediction and Evaluation Model of Physical Training Effect}

We utilize grey Markov theory to construct a prediction and evaluation model of physical training effect for volleyball players. The architecture is illustrated in Figure 2.

This section is organized as follows. In Section 3.1, the theoretical basis for predicting and evaluating the physical training is discussed. In Section 3.2, the information sampling and feature analysis of training effect is discussed. Finally, in Section 3.3, prediction and the construction of evaluation model is discussed.

3.1. Theoretical Basis. Both grey theory and Markov theory can be used for the prediction and evaluation of timeseries problems. The advantage of grey theory lies in short-term forecasting, but its disadvantage is the poor fitting of long-term forecasts and large volatility data series. The advantage of Markov theory lies in long-term prediction of data series with large random volatility, which can just make up for the limitations of grey theory. However, the prediction of Markov theory is required not only to have the characteristics of Markov chain but also to have the characteristics of the mean value of the stable process. Most of the physical training activities are nonstationary random processes that show a certain trend of change over time. The mechanism of Markov chain is shown in Figure 3.

By combining the grey theory and Markov theory, we can formulate a grey Markov physical training effect prediction and evaluation model that can reveal the general trend of physical training and development. The newly built model not only reflects the development trend of the data sequence but also reduces the range of prediction interval via the transformation of state transition probability matrix. Therefore, the prediction is more adaptable and the prediction accuracy is higher.

\subsection{Information Sampling and Feature Analysis of Physical} Training Effect. In order to realize the optimization design of the athlete's physical training effect evaluation model based on the Markov model, the big data feature analysis method is used to carry out the adaptive optimization of the their training effects. In doing so, the adaptive fusion parameter analysis model for the evaluation of the athlete's physical training effect is established. The method of data analysis and feature scheduling is used to sample the big data information of the athlete's training effect and evaluation, combined with the statistical information mining method to evaluate the effect of their physical training [35]. It can be formulated as follows:

$$
X^{(0)}=\bigcup_{i=1}^{N} x^{(i)} .
$$

The similarity feature's analysis method is used to carry out statistical analysis and optimization evaluation of the athletes' physical training effect and evaluation. The multiple regression test analysis method is adopted to establish the fuzzy constraint parameter analysis model of their training effects. The fuzzy statistical analysis and quantitative game method are used to carry out the athletes' physical training. The adaptive learning of the evaluation, the establishment of a quantitative analysis model for the evaluation of athlete's physical training, and the statistical function for the evaluation of the athlete's physical training effect is calculated as follows:

$$
\begin{aligned}
& \min F=R^{2}+A \sum_{i} \xi_{i}, \\
& \left\|\varphi\left(x_{i}\right)-o\right\|^{2} \leq R^{2}+\xi_{i} .
\end{aligned}
$$

This equation aims to combine the autocorrelation feature matching method for the fusion processing of athletes' physical training and improve the adaptability of evaluating their training effects.

Next, the statistical analysis of mechanical parameters and the method of big data sampling are combined, and big data fusion processing of these parameters is carried out. The descriptive statistical sequence of the athlete's physical training effect is depicted and formulated as follows: 


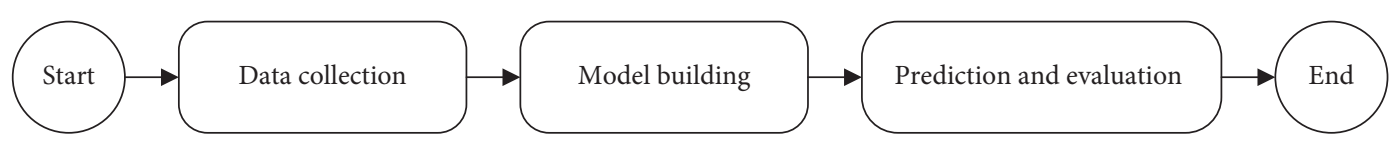

FIGURE 2: The overall architecture of our prediction and evaluation model.

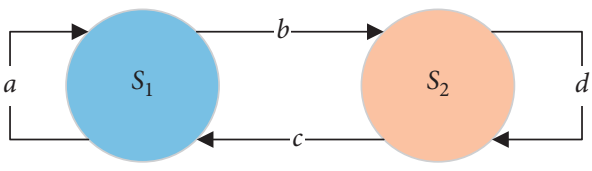

Figure 3: The mechanism of Markov chain.

$$
x_{i}=x\left(t_{0}+i \nabla t\right), \quad i=0,1, \ldots, N-1 \text {. }
$$

The quantified set of optimized feature parameters for athletes' physical training is

$$
X=\left[s_{1}, s_{2}, \ldots, s_{k}\right]_{n} .
$$

Next, an evaluation model of athlete's physical training effect based on the joint analysis of physical endurance and explosive power characteristics is designed and combined with statistical data and big data sampling methods to analyze the evaluation parameters of athletes' physical training and a fuzzy parameter fusion model for the evaluation is established. The expression of the statistical analysis model for constructing the evaluation of the physical fitness training effect of big athletes is

$$
\frac{\mathrm{d} z(t)}{\mathrm{d} t}=F(t)
$$

We use mechanics sensors to collect explosive physical data of athletes' physical training by designing a parameter distribution model and performing associated scheduling and ambiguity feature analysis. A distribution model of inertial moments of athletes' physical training explosives is designed and combined with fuzzy information fusion feature extraction to perform distributed reconstruction of the mechanical characteristics of athletes' physical training. The distribution of mechanical characteristics of athletes' physical training is as follows:

$$
\lambda=\frac{1}{\left(1+\alpha(\partial s / \partial t)^{2}\right)} .
$$

\subsection{Physical Training Effect Prediction and Evaluation Model} Construction. To understand the future grey prediction through processing of original data sequence and the establishment, learning, discovery, and mastering of the grey model, we make a scientific and quantitative prediction of the future state of the system. It is mainly used to fit and predict the eigenvalues of a dominant factor in a complex system, to reveal the changing law and the future development trend.

For data sequence test before grey model prediction, it is necessary to perform a grade comparison test on the original data sequence. The grade ratio test is formulated using

$$
\alpha(k)=\frac{x^{(0)}(k-1)}{x^{(0)}(k)}, \quad k \geq 2 .
$$

If $\alpha(k) \in\left(e^{-(2 /(n+1))}, e^{2 /(n+1)}\right)$, it is considered that the data sequence can be used to model and predict grey. In case of athletes, if any athlete does not meet this condition, then certain processing needs to be performed on the original data sequence to meet the conditions of grade ratio test. For this purpose, the data transformation is usually used. There are translation exchange methods, logarithmic transformation method, and square root transformation method, as well for this purpose. Next, we accumulate the original time data series as follows:

$$
X_{(t)}^{(1)}=\left(x^{(1)}(1), x^{(1)}(2), \ldots, x^{(1)}(n)\right) .
$$

Generate sequence $X_{(t)}^{(1)}$ with equal weights adjacent to $Z_{(t)}^{(1)}$ as follows:

$$
\begin{aligned}
Z_{(t)}^{(1)} & =\left(z^{(1)}(1), z^{(1)}(2), \ldots, z^{(1)}(n)\right), \\
z^{(1)}(1) & =x^{(1)}(1), \quad k=1, \\
z^{(1)}(k) & =0.5 x^{(1)}(k)+0.5 x^{(1)}(k-1), \quad k>1 .
\end{aligned}
$$

The first-order univariate differential equation is used to fit and model the generated data sequence, and the grey prediction model is obtained as follows:

$$
\frac{\mathrm{d} x^{(1)}}{\mathrm{d} t} a s+a x^{(1)}=b,
$$

where $a$ is the development parameter and $b$ is the grey effect.

Please note that the parameter column $\bar{a}=(a, b)^{T}$ is determined using the least square method:

$$
\begin{aligned}
\widehat{a} & =\left(B^{T} B\right)^{-1} B^{T} Y_{n}, \\
B & =\left[\begin{array}{cc}
-z^{(1)}(2) & 1 \\
-z^{(1)}(3) & 1 \\
\ldots & \ldots \\
-z^{(1)}(n) & 1
\end{array}\right], \\
Y_{n} & =\left[\begin{array}{c}
x^{(0)}(2) \\
x^{(0)}(3) \\
\cdots \\
x^{(0)}(n)
\end{array}\right] .
\end{aligned}
$$

Next, we find the solution of the whitening equation, that is, the time response sequence using 


$$
\bar{x}^{(1)}(k+1)=\left(x^{(0)}(1)-\frac{a}{b}\right) e^{-a k}+\frac{b}{a} .
$$

To restore the value data sequence by accumulative subtraction generation method, we use

$$
\bar{x}^{(0)}=\left(\bar{x}^{(0)}(1), \bar{x}^{(0)}(2), \ldots, \bar{x}^{(0)}(n)\right) .
$$

The Markov process is based on the state of each data in the existing data sequence and the state transition law constructed by the data sequence, predicting that a certain data system may appear in a certain state within a period of time in the future. To provide theoretical support for related decision management, the basic model of the Markov forecasting method is used as shown in

$$
x(k+1)=x(k) \times P .
$$

The change process of physical fitness is an unstable random process that randomly presents an upward or downward trend. The physical fitness data sequence conforms to the $n$-order Markov nonstationary random sequence. Take $\widehat{Y}(k)=\widehat{x}^{(0)}(k+1)$ as the baseline; according to the actual prediction of the physical fitness value, the physical fitness prediction sequence can be divided into several interval values, i.e., several states, as shown in equations (15)-(18):

$$
\begin{aligned}
Q_{1 i} & =\left[Q_{1 i}, Q_{2 i}\right], \\
Q_{1 i} & =\widehat{Y}(k)+A_{i}, \\
Q_{2 i} & =\widehat{Y}(k)+B_{i} .
\end{aligned}
$$

The number of original data samples from state $Q_{i}$ to state $Q_{j}$ after $n$-step transition is denoted as $M_{i j}^{(n)}$, where $M_{i}$ is the frequency of state $Q_{i}$; then, the probability that a state reaches state $Q_{j}$ after $n$-step transition is

$$
P_{i j}^{(n)}=\frac{M_{i j}^{(n)}}{M_{i}} .
$$

The state transition probability matrix is obtained using

$$
P_{i j}^{(n)}=\left[\begin{array}{llll}
P_{11}^{n} & P_{12}^{n} & \ldots & P_{1 n}^{n} \\
P_{21}^{n} & P_{22}^{n} & \ldots & P_{2 n}^{n} \\
& & \ldots & \\
P_{n 1}^{n} & P_{n 2}^{n} & \ldots & P_{n n}^{n}
\end{array}\right] .
$$

Through the obtained one-step state transition probability matrix, the current initial state distribution can be determined, and the state or development trend that may appear in the future can be predicted. The state transition probability matrix reflects the transition law between various states in the system. Moreover, it can determine the turn of the future state.

When the future direction of the system is determined, the change range of its grey function is determined as $\left[Q_{1 i}, Q_{2 i}\right]$. Since $Q_{1 i}$ and $Q_{2 i}$ are the lower critical value and upper critical value of a certain state area, respectively, the interval average value is taken as the predicted value in the future. The calculation formula is

$$
Y^{\prime}(k)=0.5\left(Q_{1 i}+Q_{2 i}\right) .
$$

According to equations (16) and (17), equation (20) can be transformed as follows:

$$
Y^{\prime}(k)=\widehat{Y}(k)+0.5\left(A_{i}+B_{i}\right) .
$$

To further improve the accuracy of the model, in future predictive modeling, this article first uses the metabolic method in the grey theory model to process the original data and then performs grey Markov modeling and prediction. In the modeling process, the data sequence is divided into many states, and the middle value of the grey element interval is taken as the final prediction value to achieve higher prediction accuracy. If the state of the division is small, a relatively conservative prediction principle can be used, and the grey element interval can be adopted. The lower value of the critical value is used as the predicted value.

\section{Model Testing and Discussion}

It is impossible for any prediction model to be $100 \%$ accurate. Hence, whether the prediction of the grey Markov model is reliable requires a quantitative test for accuracy. This article not only uses residual test and posterior test but also uses the grey system theory analysis method, and model testing, testing the relevance of the original data series and the predicted data series. The classification of accuracy testing is detailed in Table 1:

To verify the performance of the proposed method for the realization of athletes' physical training effects, simulation test analysis was carried out. SPSS 14.0 statistical analysis software was used to analyze the constraint parameters of athletes' physical training effects and statistical analysis, and mechanical sensors were used to evaluate the effects of athletes' physical training. The statistical analysis results of physical information collection and physical training effect are shown in Table 2.

According to the data of Table 2, the development parameters and the grey effect are obtained, and then, they are substituted into the definition of grey prediction. A model is obtained for predicting values and residuals of the first five volleyball athletes' physical training performance, as shown in Table 3.

It can be seen from the experimental results that the prediction error is small and within an acceptable range, which shows the validity of the model. Since the fifth prediction is not the optimal state, the actual value is used as the initial state, and the seventh physical fitness prediction and correction is carried out. It is found out that the sixth prediction effect is better. The comparison result with GM is shown in Table 4. In this table, "AV" is the actual value, "PV" is the predictive value, "RR" is the relative residual, and "RV" is the revised value.

It can be seen from Table 4 that the accuracy of the GM $(1,1)$ model is significantly improved after the grey-scale Markov chain is corrected, and the relative error is reduced. 
TABLE 1: Accuracy inspection: grade reference table.

\begin{tabular}{lcc}
\hline Accuracy level & Residuals & Relative residuals \\
\hline Excellent & 0.35 & 0.01 \\
Good & 0.50 & 0.05 \\
Qualified & 0.65 & 0.10 \\
Unqualified & 0.80 & 0.20 \\
\hline
\end{tabular}

TABLe 2: Statistical analysis for evaluating physical fitness training effect.

\begin{tabular}{lcccc}
\hline Variable & Mean & Standard value & Minimum & Statistical average \\
\hline Exercise time & 0.265 & 0.675 & 0.136 & 5.701 \\
Physical fitness & 0.363 & 0.534 & 0.355 & 2.455 \\
Daily exercise & 0.549 & 0.422 & 0.451 & 2.565 \\
Training intensity & 0.450 & 0.470 & 0.373 & 4.536 \\
Correlation coefficient & 0.437 & 0.538 & 0.429 & 3.612 \\
\hline
\end{tabular}

TABLE 3: Comparison of actual and predicted values of first five physical training.

\begin{tabular}{lcccc}
\hline Order & $\begin{array}{c}\text { Actual } \\
\text { value }\end{array}$ & $\begin{array}{c}\text { Predictive } \\
\text { value }\end{array}$ & Residual & $\begin{array}{c}\text { Relative residual } \\
(\%)\end{array}$ \\
\hline 1 & 25.7 & 25.7 & 0 & 0 \\
2 & 24.9 & 24.6 & 0.2 & 0.803 \\
3 & 25.7 & 25.9 & -0.2 & -0.778 \\
4 & 23.6 & 23.3 & 0.3 & 1.271 \\
5 & 23.9 & 23.2 & 0.7 & 2.929 \\
\hline
\end{tabular}

TABle 4: The sixth training performance prediction value and correction value.

\begin{tabular}{lccccccc}
\hline \multirow{2}{*}{ Order } & \multirow{2}{*}{ AV } & \multicolumn{3}{c}{ GM $(1,1)$} & \multicolumn{3}{c}{ Grey Markov model } \\
& & PV & $R$ & RR (\%) & RV & $R$ & RR (\%) \\
\hline 7 & 24.8 & 24.1 & 0.7 & 2.823 & 24.5 & 0.3 & 1.210 \\
\hline
\end{tabular}

The grey prediction model alone has a higher relative error. It can be seen that the accuracy of the grey Markov prediction model is obviously higher than that of the grey prediction model GM $(1,1)$ alone.

According to the descriptive statistical analysis results of the evaluation of athlete's physical training effect in Table 2, the evaluation of the physical training effect is carried out, and the collected results of the mechanical parameters of the athlete's physical training is obtained. Using the results of collected mechanical parameters of athlete's physical training in Table 2, we can evaluate the effect of athlete's physical training. The optimized output of the evaluation is shown in Figure 4.

Analyzing Figure 4, we know that the method in this paper has high accuracy in evaluating athletes' physical training effects, better feature tracking performance, and confidence in test evaluation. Compared with some similar methods [36] and [37], the comparison result is shown in Figure 5.

It can be seen that the model we proposed has strong competitiveness in terms of physical fitness prediction and assessment.

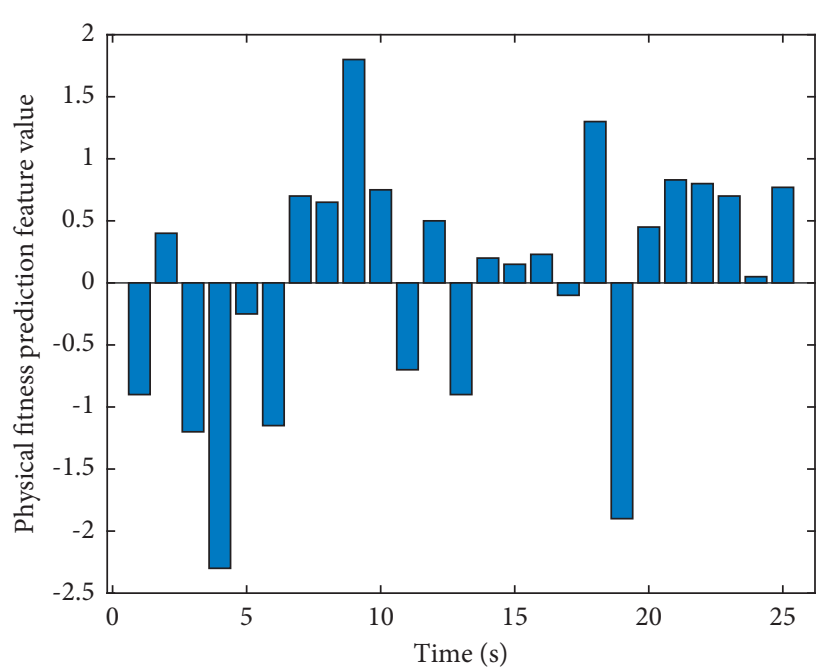

FIgURE 4: Athlete's physical training effect evaluation output.

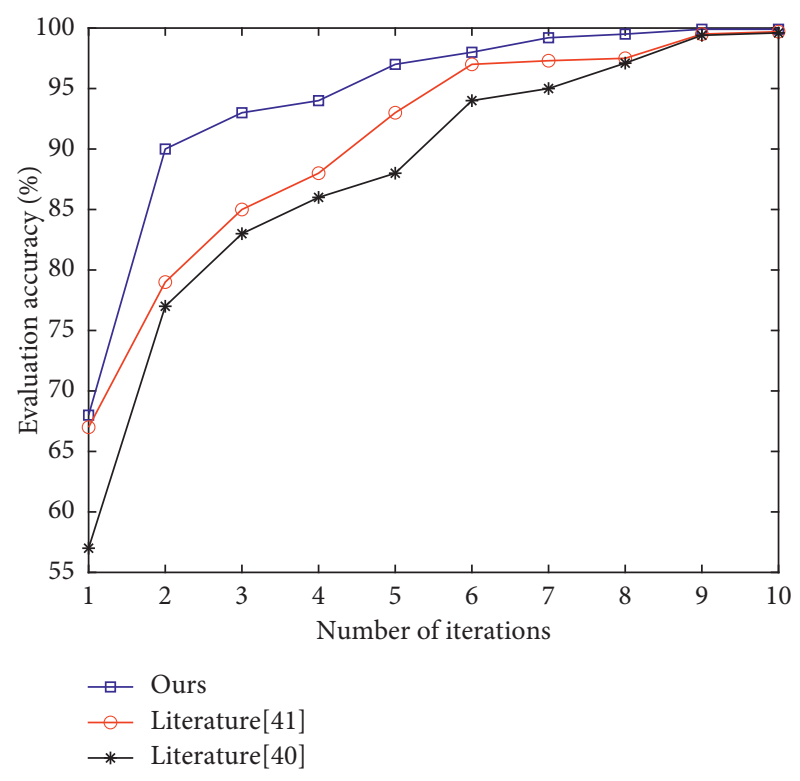

Figure 5: Evaluation accuracy comparison test. 


\section{Conclusions}

In this paper, the grey Markov model is used to predict and evaluate the physical fitness changes of volleyball players. Initially, the players' training performance in each state is evaluated using the Markov model. The statistical results and evaluation parameters are combined to form an empirical model for training. The calculation of the model is relatively simple and the accuracy is much higher. The grey prediction model is mainly suitable for system objects with short prediction time and little fluctuation. Due to its own shortcomings, the proposed work can only be applied to systems where the original data sequence changes exponentially. The Markov model is suitable for predicting dynamic processes with large random fluctuations. At this point, it can make up for the shortcomings of grey prediction model to achieve complementary functions, highlight advantages, and improve the prediction accuracy. In the future, I aim to work on different models (other than Markov) for the prediction and evaluation of physical training of volleyball players to see the effects and efficiency.

\section{Data Availability}

The datasets used are available from the author upon reasonable request.

\section{Conflicts of Interest}

The author declares that he has no conflicts of interest.

\section{Acknowledgments}

This project was supported by the 2021 Support Program for Outstanding Young Talents in Universities in Anhui Province, project no. gxyq2021139 (construction of integration of physical education curriculum in higher vocational colleges from the perspective of subject core literacy).

\section{References}

[1] F. Yan and C. Li, "Cause analysis on the prominence of body capability in present sports training and competition," Journal Of Beijing Teachers College Of Physical Education, vol. 12, no. 3, pp. 27-30, 2000.

[2] D. Lian, S. Huang, and J. Su, "The analysis on present situation of physical ability of elite male volleyball players in China," China Sport Science and Technology, vol. 37, no. 6, pp. 16-18, 2001.

[3] W. Zhang, "Innovative development of sports science of volleyball," Insight-Sports Science, vol. 2, no. 2, 2020.

[4] M. Yu, J. Jin, X. Wang, X. Yu, D. Zhan, and J. Gao, "Development and design of flexible sensors used in pressuremonitoring sports pants for human knee joints," IEEE Sensors Journal, p. 1, 2021.

[5] X. Yu, D. Zhan, L. Liu, H. Lv, L. Xu, and J. Du, "A privacypreserving cross-domain healthcare wearables recommendation algorithm based on domain-dependent and domainindependent feature fusion," IEEE Journal of Biomedical and Health Informatics, p. 1, 2021.

[6] M. Marra, O. Di Vincenzo, R. Sammarco et al., "Bioimpedance phase angle in elite male athletes: a segmental approach. Physiological Measurement," vol. 41, no. 12, Article ID 125007, 2020.

[7] R. Zhang, "Features of the women volleyball player' s body shape and bounce quality in the 26th olympic game -analyzing the present situation of asian women volleyball teams," Journal of Guangzhou Physical Education Institute, vol. 18, no. 1, pp. 99-103, 1998.

[8] S. Ren and Q. Zeng, "Feature in hemodynamic index changes of shanghai male volleyball players under different states," Journal of Shanghai Physical Education Institute, vol. 23, no. 1, pp. 61-66, 1999.

[9] B. Liu, "The characteristics of energy supply in volleyball matches and the training to improve energy supply," China Sport Science and Technology, vol. 33, no. 3, 1997.

[10] W. Song and L. Du, "Strength training of volleyball player," China Sport Science and Technology, vol. 34, no. 9, pp. 34-36, 1998.

[11] Z. Gao, F. Gao, and X. Wang, "The dynamic characteristics in research on strength training and its training methods in modern volleyball," Sports Sciences Researches, vol. 4, no. 3, pp. 55-59, 2000.

[12] J. Wu, "Diagnosis and analysis on volleyball specialized sport injury," Bulletin of Sport Science \& Technology, vol. 7, pp. 81-83, 2000.

[13] X. Yu, F. Jiang, J. Du, and D. Gong, "A cross-domain collaborative filtering algorithm with expanding user and item features via the latent factor space of auxiliary domains," Pattern Recognition, vol. 94, pp. 96-109, 2019.

[14] J. Pei, "Big data mining in the control of epidemic," Basic and Clinical Pharmacology and Toxicology, vol. 126, pp. 428-430, 2020.

[15] X. Feng, T. Yang, L. Sun, T. Ma, and Y. Hua, "Influence of functional dynamic tensile training on men's football players' FMS and sports injury rate," Sport Science Research, vol. 32, no. 5, pp. 33-36, 2011.

[16] B. Mou, "Analysis of Taizhou city youth wushu athletes' functional motion test," Hubei Sports Science, vol. 31, no. 5, pp. 611-612, 2012.

[17] Y. Fu, "Analysis of shenzhen canoe athletes' body function through action test," Hubei Sports Science, vol. 31, no. 3, pp. 369-370, 2012.

[18] K. I. Minick, K. B. Kiesel, L. Burton, A. Taylor, P. Plisky, and R. J. Butler, "Interrater reliability of the functional movement screen," The Journal of Strength \& Conditioning Research, vol. 24, no. 2, pp. 479-486, 2010.

[19] A. Schneiders, S. Davidsson, E. Hrman, and S. Sullivan, "Functional movement screentm normative values in a young," Active Population, International Journal of Sports Physical Therapy, vol. 6, no. 2, 2011.

[20] J. A. Onate, T. Dewey, R. O. Kollock et al., "Real-time intersession and interrater reliability of the functional movement screen," The Journal of Strength \& Conditioning Research, vol. 26, no. 2, pp. 408-415, 2012.

[21] L. Sun and M. Li, "Sports and health management using big data based on voice feature processing and Internet of things," Scientific Programming, vol. 2021, Article ID 3271863, 10 pages, 2021.

[22] W. Guo and Y. Niu, "Interactive teaching system of basketball action in college sports based on online to offline mixed teaching mode," Mobile Information Systems, vol. 2021, 2021.

[23] J. Zhuang, J. Sun, and G. Yuan, "Arrhythmia diagnosis of young martial arts athletes based on deep learning for smart medical care," Neural Computing and Applications, pp. 1-12, 2021. 
[24] L. Meng and E. Qiao, "Analysis and design of dual-feature fusion neural network for sports injury estimation model," Neural Computing and Applications, pp. 1-13, 2021.

[25] R. W. Bisseling, A. L. Hof, S. W. Bredeweg, J. Zwerver, and T. Mulder, "Are the take-off and landing phase dynamics of the volleyball spike jump related to patellar tendinopathy?" British journal of sports medicine, vol. 42, no. 6, pp. 483-489, 2008.

[26] Z. Fu and Y. Cheng, "Status Quo of Research on Volleyball Players' Physical Training," Sports Technology Information, vol. 2, no. 20, pp. 43-47, 2000.

[27] M. Wang, "Measurement and Evaluating of Elite Male youth volleyball Athletes' Physical Fitness," Journal of PLA Institute of Physical Education, vol. 23, no. 3, pp. 55-56, 2004.

[28] S. Chen, "Comparative Study on the Evaluating Criteria and the Results of the Field Work of Students' Physical Health (Physical Fitness) Between Fujian and Taiwan," Fujian Sports Science and Technology, vol. 30, no. 5, pp. 4-6, 2011.

[29] J. Yang, "How to Measure the Physical Fitness of American Volleyball Players," Chinese Volleyball, vol. 1995, no. 2, pp. 4-8, 1995.

[30] L. Zhang, F. Xu, and W. Zhao, "Study of Drawing Up Standards of Appraising Physique of National Major Women's Volleyball," Journal of Xi'an Institute of Physical Education, vol. 13, no. 1, p. 6, 1996.

[31] H. Feng and M. Mei, "A Study on the Test Items and Evaluation Model of the Specific Physical Fitness of Junior Group B Volleyball Players in my country," Journal of Nanjing Institute of Physical Education, vol. 8, no. 4, pp. 47-48, 2009.

[32] B. Yang, B. Cheng, Y. Liu, and L. Wang, "Deep learningenabled block scrambling algorithm for securing telemedicine data of table tennis players," Neural Computing and Applications, pp. 1-14, 2021.

[33] R. Yi, L. Tang, Y. Tian, J. Liu, and Z. Wu, "Identification and classification of pneumonia disease using a deep learningbased intelligent computational framework," Neural Computing and Applications, pp. 1-14, 2021.

[34] F. Jiang and X. Chen, "An Action Recognition Algorithm for Sprinters Using Machine Learning," Mobile Information Systems, vol. 2021, 2021.

[35] G. Song and J. Wu, "Influence of Siberian Ginseng P. E. on the training effects of anaerobic exercise," Journal of Huaqiao University, vol. 36, no. 5, pp. 558-562, 2015.

[36] K Zhong, Y Wang, J Pei et al., "Super efficiency SBM-DEA and neural network for performance evaluation[J]," Information Processing \& Management, vol. 58, no. 6, Article ID 102728, 2021.

[37] Y. Hu, W. Wu, J. Li, and W. Tao, "New Exercise System for Waist and Back and Its Effect Detection," Journal of Nanjing University of Science and Technology, vol. 40, no. 6, pp. 734-739, 2016. 